\title{
ASPECT - Insufficient Care Environment Regarding Privacy, Views, Comfort and Facilities for Critically III Patients at One Emergency Department in Vietnam - An Observation Study
}

\author{
Sara Charmi, Amanda Johansson Landell and Kristina Rosengren* \\ Sahlgrenska Academy, Institute of Health and Care Sciences, University of Gothenburg, Sweden
}

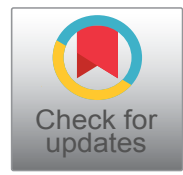

*Corresponding author: Kristina Rosengren, PhD, RN, Associate Professor, Sahlgrenska Academy, Institute of Health and Care Sciences, University of Gothenburg, Sweden, ORCID iD: 0000-0002-3221-2062

\begin{abstract}
The importance of a healthy environment was noted by Florence Nightingale in the $19^{\text {th }}$ century and is part of nurses' role of increasing health and well-being. The aim of this study was to investigate the care environment in emergency care (emergency ward and intensive care unit) at Hanoi Medical University Hospital in Vietnam. The study was conducted in the emergency department of a teaching hospital in Vietnam using a mixed methods design; quantitative (standardized protocol) and qualitative (field notes) methods with an inductive approach. The data were collected through ASPECT $(n=652)$ and field notes ( 9 A4 pages), which were analysed using descriptive statistics and qualitative content analysis. The results showed an inadequate care environment, especially with regard to patients' privacy. In the emergency ward, most patients shared beds, compared to the intensive care unit (ICU). Moreover, the ICU lacked access to fresh air and had indoor views. Increased awareness is needed among caregivers and decision makers regarding the significance of a healthy environment to improve the quality of care. A healthy environment can help to improve hospitals with crowded rooms and issues with odour, cleanness, temperature, and limited water and meal services.
\end{abstract}

\section{Keywords}

ASPECT, Emergency department, Healthy environment, Improvement, Mixed methods, Vietnam

\section{Introduction}

In Vietnam, governmental health insurance covers part of the costs of healthcare, but patients and relatives must pay the majority of healthcare expenses [1]. Limited resources within Vietnamese healthcare influences patients' and their relatives' experiences of healthcare, as do gender and localization [2]. Overall, patients rate their satisfaction rather low and describe dissatisfaction regarding the lack of beds and limited space within the stressful and overcrowded hospital environment [3,4]. According to Nightingale [5], five environmental aspects are important: Ventilation, clean water, an effective sewage system, and clean and bright rooms. Therefore, this study examines the hospital environment in an attempt to improve the caring environment in Vietnam.

\section{Background}

Healthcare in Vietnam involves overloaded hospitals (both public and private), especially smaller hospitals with limited equipment and less competent staff members. Resources are needed to upgrade older medical devices to improve the quality of care. Vietnam has approximately 90 million inhabitants, and its transition to a market economy improved living conditions for the population. Common infectious diseases in Vietnam include dengue fever, HIV and measles, and well-known risk factors are alcohol, tobacco and traffic injuries. The average life expectancy in 2009 was 75.6 years for females and 70.2 years for males, and the highest rates of mortality and disease burden were attributed to tuberculosis, malaria, cancer, and chronic respiratory and cardiovascular diseases $[6,7]$.

Given the limited resources and quality within

Citation: Charmi S, Landell AJ, Rosengren K (2018) ASPECT - Insufficient Care Environment Regarding Privacy, Views, Comfort and Facilities for Critically III Patients at One Emergency Department in Vietnam - An Observation Study. Int Arch Nurs Health Care 4:112. doi.org/10.23937/2469-5823/1510112 Accepted: December 11, 2018: Published: December 13, 2018

Copyright: (c) 2018 Charmi S, et al. This is an open-access article distributed under the terms of the Creative Commons Attribution License, which permits unrestricted use, distribution, and reproduction in any medium, provided the original author and source are credited. 
Vietnamese healthcare, patients often choose private hospitals [2,8]. Research [4] shows patients' dissatisfaction regarding the quality of care in relation to the care environment, including crowded rooms, odour, heat, and a lack of water and meal services. Improvement is needed, such as single-bed rooms and clean toilets $[2,4,8]$. Due to these shortcomings as well as a lack of resources, health has become a matter of class due to the economic burden among patients and relatives. Nurses can influence the care environment [1], and the adaptation of the care environment to improve health and well-being is a significant part of nursing [9]. This idea is in line with Nightingale's [5] experiences regarding a healthy caring environment and patient health. Soldiers did not die in hospital due to war injuries; instead, they died due to contaminated water or shortcomings in the sewage systems (i.e., the healthcare environment). Nightingale [5] noted that ventilation with access to fresh air and bright rooms with windows (view, air, sunlight) are needed to improve the environment, as are the regulation of temperature and curtains around beds in hospitals. There is an emotional and concrete link between clean and comfortable beds, noise reduction (to improve sleep and concentration), and colours and shapes (which have physical and mental effects) and decreased pain [5]. Memories due to experiences in the hospital environment affect health (appetite, well-being); this is why freshness (i.e., odour influences well-being in a negative way) is highlighted to reduce anxiety in acute situations [10]. According to the International Council of Nurses (ICN) [9], significant components of nursing to promote health and prevent illness include infection control, risk management, safe drug use, and good standards within the care environment. However, there are still limitations due to the hospital environment [10]. Therefore, the aim of this study was to investigate the care environment in emergency care (emergency ward and intensive care unit, ICU) at one private teaching hospital in Vietnam.

\section{Research Method}

The study was conducted in the emergency department of a teaching hospital in Vietnam with 350 beds, 600 students, 320 staff members and 72 physicians $[11,12]$. The emergency ward (20 beds) consisted of an entrance, reception and triage room, including a trauma room ( 3 beds), a room for febrile patients ( 3 beds) and a general room (14 beds). The ICU ( 9 beds) consisted of an entrance, reception, two single rooms and two other rooms (three and four beds).

This study used a mixed methods design as well as quantitative (standardized protocol) and qualitative (field notes) methods with an inductive approach to increase understanding of the care environment in the emergency department. A standardized observation protocol, A Staff and Patient Environment Calibration Toolkit (ASPECT) [13], was used, thereafter a pilot study (12 observations) was conducted at four wards in Sweden to assess the protocol (empirical grounded methodological justification). No changes were made before data collection was initiated in Vietnam. ASPECT [13] includes 47 items within eight areas: C1: Privacy, company and dignity, C2: Views, C3: Nature and outdoors, C4: Comfort and control, C5: Legibility of place, C6: Interior appearance, C7: Facilities, and C8: Staff. A six-point Likert scale (1. Virtually no agreement, 2. Hardly any agreement, 3. Little agreement, 4. Fair agreement, 5. Strong agreement, and 6. Virtually complete agreement), descriptive statistics [14] and content analysis [15] were used to develop knowledge that is trustworthy with respect to validity and reliability as well as credibility, dependability and transferability.

A strategic sample was facilitated by a contact person at the nursing programme at Hanoi Medical University. The sample included two wards, an emergency ward and an ICU at one private teaching hospital in Vietnam. One hospital manager was contacted regarding interest in participating. After agreement, staff members at the emergency department were informed about the study's aim and procedures, and voluntary participation and confidentiality were assured. Data collection was conducted in two wards (an emergency and an intensive care unit) in October 2016. It included observations (n = 652) and field notes (nine A4 pages) regarding the number of patients in the same room/bed, aspects of cleanliness and the possibility of privacy. Two of the authors (SC and AJL) observed different rooms for six days due to limited timetable for nursing student to conduct a bachelor thesis. Five places were observed in the emergency ward: the entrance, triage, and febrile, trauma and general rooms. In the ICU, the following places were observed: the entrance, single room (x 2), and multiple room ( $x 2$ ). Variables $\mathrm{C} 1, \mathrm{C} 2, \mathrm{C} 3, \mathrm{C} 4$, and $\mathrm{C} 6$ and two variables from $\mathrm{C} 8(8.02,8.05)$ were observed in all rooms. C5, C7 and three variables from C8 (8.01, $8.03,8.06)$ were observed only once, although these variables were interpreted as general for the hospital as a whole. A total of 163 variables were observed. Each observation lasted 60 minutes and was conducted during weekdays as well as weekends, including morning and afternoon shifts [14].

The quantitative data (protocol, $n=652$ ) were analysed using descriptive statistics via Microsoft Office Excel [16]. Weighting regarding the quality of the care environment was determined as high (2), normal (1) or zero (0) to determine the effect of the statements for an overall score. A statement with high importance was given a weighting of 2 (instead of the normal 1) (i.e., a double effect). For example, privacy is more important in patient rooms than in the entrance, so a single room in an ICU could score 6 on C1: Privacy, company and dignity, which were added twice (weighting). The results were doubled, which is why the mean of this variable was increased. The overall mean for the emergency 
Table 1: C1: Privacy, company and dignity.

\begin{tabular}{|l|l|l|l|l|l|}
\hline Emergency ward & 1.01 & 1.02 & 1.03 & 1.04 & 1.05 \\
\hline Entrance & & & & & \\
\hline Triage & & & & & \\
\hline Trauma & & & & & \\
\hline Febrile & & & & & \\
\hline General room & & & & & \\
\hline Intensive care unit & 1.01 & 1.02 & 1.03 & 1.04 & 1.05 \\
\hline Entrance & & & & & \\
\hline Single room 1 & & & & & \\
\hline Single room 2 & & & & & \\
\hline General room 1 & & & & & \\
\hline General room 2 & & & & & \\
\hline
\end{tabular}

Note: Green indicates the highest score (5-6), yellow indicates a moderate score (3-4) and red indicates the lowest score (1-2).

ward and the ICU were also calculated (all data divided by 47$)$, and the mean was calculated for all variables. In addition, analysis (average) of each observation was performed and given a colour, as reported in the tables in the results. Green indicates the highest score (5-6), yellow indicates a moderate score (3-4) and red indicates the lowest score (1-2). All data were calculated (multiplied or divided; for example, 13/6 = $2.16666666666666=2.167$ ) to show the percentages of the identified similarities and differences.

Field notes (nine A4 pages) were analysed using manifest qualitative content analysis [15] with a step-by-step procedure. Written words from the data (field notes) were used for the analysis. The texts were read to acquire an initial impression of the content concerning compliance with ASPECT's [13] eight areas. The analysis addressed questions regarding the caring environment in emergency care and was performed in the following steps. (1) The written words were read and re-read to obtain an understanding of and familiarity with the text regarding ASPECT's [13] eight areas. (2) Meaning units (words, sentences or paragraphs) corresponding to the content areas were selected for (a) Limited space and (b) Facilities. (3) Each meaning unit was condensed into a description of caring aspects regarding the influence of the hospital environment. (4) Content was identified and clustered into ASPECT's [13] eight areas.

Ethical approval and permission for the study were obtained from the director of the Nursing Department, Hanoi Medical University. No ethical approval was needed due to Swedish rules and guidelines regarding a student's thesis and/or quality improvement that has no negative effects for the participants $[17,18]$. However, ethical guidelines for human and social research were followed throughout the study [17]. Therefore, respect for the individual (staff, patients, relatives) was a main concern during the study with regard to the observation of the hospital environment in emergency care. For example, no individuals participated in the study, although the observation focused on the caring environment with regard to ASPECT's [13] eight areas. All staff, patients and relatives who were involved in caring activities during the observation were informed about the aim of the observations and were assured that every observation was voluntary and confidential. Therefore, the results are described in categories in relation to ASPECTs eight areas without identification to any person (staff, patients and relatives) to maintain respect for integrity and confidentiality [17].

\section{Findings}

The results are presented in two parts, tables and the body text regarding similarities and differences between the emergency ward and the ICU based on ASPECT's [13] eight areas: C1: Privacy, company and dignity, C2: Views, C3: Nature and outdoors, C4: Comfort and control, C5: Legibility of place, C6: Interior appearance, C7: Facilities, and C8: Staff. In addition, the results are presented by number (for example, 1.01 (1 represents $\mathrm{C} 1$, and the 01 represents item 1 ) as well as different colours depending on the score (red $=1-2$ points, yellow $=3-4$ points, green $=5-6$ points). Two variables $(8.02$, $8.05)$ in $\mathrm{C} 8$ were observed in all places in the wards, whereas $C 5, C 7$ and three variables $(8.01,8.03,8.06)$ in C8 were observed once at the wards as these results were interpreted as general for the hospital. Part two includes field notes grouped with regard to similarities and differences related to $\mathrm{C} 1-\mathrm{C} 8$, described in the body text (number of patients in rooms or bed, cleanliness and possibility of privacy in patient rooms). The average for the emergency ward was 1.7 compared to 1.8 for the ICU; that is, the ICU had generally higher quality in the care environment than the emergency care ward due to different strengths and deficiencies. For example, in the general room at the emergency ward, twenty patients were treated within 15 beds (i.e., most patients shared beds), compared with the ICU, where no patients shared beds. The possibility of privacy was limited due to shared beds as well as more space for health professionals to perform undisturbed caring activities. The emergency ward had access to daylight from windows with outdoor views, whereas the ICU had windows showing inside views of the hospital corridors.

\section{C1: Privacy, company and dignity}

Neither the emergency ward nor the ICU had waiting rooms, so patients and relatives had to wait outside the ward area (Table 1). In the emergency ward, patients and relatives must wait outside the hospital building, so the outdoor environment is more important than it is in the ICU, where patients and relatives wait in a hospital corridor. The front desk at both wards was not separated with doors; it was placed directly in relation to the caring activities. At the emergency ward, the front desk was located in the same area as triage ( 3 beds) and the general room ( 14 beds), which made the place more crowded than the front desk at the ICU, which had a lower number of beds (general room, 3 
Table 2: C2: Views

\begin{tabular}{|l|l|l|l|l|l|}
\hline Emergency ward & 2.01 & 2.02 & 2.03 & 2.04 & 2.05 \\
\hline Entrance & & & & & \\
\hline Triage & & & & \\
\hline Trauma & & & & & \\
\hline Febrile & & & & & \\
\hline General room & & & & & \\
\hline Intensive care unit & 2.01 & 2.02 & 2.03 & 2.04 & 2.05 \\
\hline Entrance & & & & & \\
\hline Single room 1 & & & & & \\
\hline Single room 2 & & & & & \\
\hline General room 1 & & & & & \\
\hline General room 2 & & & & & \\
\hline
\end{tabular}

Note: Green indicates the highest score (5-6), yellow indicates a moderate score (3-4) and red indicates the lowest score (1-2).

beds). Furthermore, there was no possibility to speak undisturbed in the wards. However, privacy may be less important in the entrance compared to the patient rooms due to the longer length of stay in a patient room.

Triage (3-bed room) was carried out at the emergency ward adjacent to the desk with a curtain/drape around the beds. Thus, isolation from sounds from the entrance was limited, and the beds were not separated from each other (no curtains/drapes), which was perceived as a messy and stressful caring environment. Patients were treated openly without the use of curtain/drapes in the ward. In response to the question of why curtain/ drapes not were used for privacy, nurses answered that "nurses choose for patients", which further reduces the patients' privacy and dignity. In addition, the presence of a window at the triage made it easy for people from other rooms to view the caring activities.

In the emergency wards, smaller rooms ( 3 beds) were equipped with curtains/drapes that offered privacy but not the ability to speak undisturbed due to shared rooms. These rooms ( $2 \times 3$ beds) were also equipped with a chair for visitors; however, there was limited space in the rooms, which complicated relatives' involvement in basic care. This is a typical situation in Vietnamese healthcare due to a lack of resources. In the general room (14 beds), there was no possibility for privacy as there were many shared beds in a small area with few curtains/drapes around the beds and a clear view from the entrance and triage, which limited patients' privacy and integrity.

The ICU had two single rooms where patients were treated individually, which increases privacy and integrity compared to the emergency ward's shared rooms. Almost every room in the ICU was equipped with doors, windows and curtains/drapes to pull around the beds. However, they were small and had limited space for relatives. One of the general rooms (4 beds) had no door but had curtains/drapes for each bed, which allowed patients to have privacy but limited the ability for private conversation. The other general room ( 3 beds) was located directly by the front desk and
Table 3: C3: Nature and outdoors.

\begin{tabular}{|l|l|l|l|}
\hline Emergency ward & 3.01 & 3.02 & 3.03 \\
\hline Entrance & & & \\
\hline Triage & & & \\
\hline Trauma & & & \\
\hline Febrile & & & \\
\hline General room & & \\
\hline Intensive care unit & 3.01 & 3.02 & 3.02 \\
\hline Entrance & & & \\
\hline Single room 1 & & & \\
\hline Single room 2 & & & \\
\hline General room 1 & & & \\
\hline General room 2 & & \\
\hline
\end{tabular}

Note: Green indicates the highest score (5-6), yellow indicates a moderate score (3-4) and red indicates the lowest score (1-2).

separated with curtains/drapes around the beds with some possibility of privacy.

In summary, privacy, company and dignity were limited; the care environment could not offer undisturbed conversation due to the limited area, especially in the emergency ward. In addition, there was a lack of space for undisturbed conversation in the entrance. However, patients and relatives spend limited time there (hours) compare to the wards (days). The emergency ward treated 20 patients in 16 beds with no single rooms, so privacy was limited because patients shared beds. In the ICU, none of the patients shared beds, which contributed to an undisturbed working and caring environment with the possibility for privacy for patients and relatives.

\section{C2: Views}

The entrance and febrile room in the emergency ward were equipped with windows with daylight viewing of a busy outdoor environment (heavy traffic) (Table 2). However, the trauma room ( 3 beds) and general room (14 beds) lacked windows. This is a shortcoming during hospitalization, when patients are in attendance for a longer period of time at the ward. The ICU had windows in all rooms with views of hospital corridors or another general room. The indoor views provided limited access to daylight as well as limited privacy. Moreover, all rooms in the ICU were adjacent to the front desk, so staff were always visible to patients and relatives. This is a significant factor that ensures safety when the staff is close to critically ill patients.

\section{C3: Nature and outdoors}

Access to nature and the outdoors were also limited (Table 3). The hospital lacked access to nature, although the outdoor areas included single trees at the hospital entrance. The ICU had no direct access to the outdoor environment compared to the emergency ward. ICU patients or relatives needed to go through the hospital or through the emergency ward to go outdoors. 
Table 4: C4: Comfort and control.

\begin{tabular}{|l|l|l|l|l|l|l|l|}
\hline Emergency ward & 4.01 & 4.02 & 4.03 & 4.04 & 4.05 & 4.06 \\
\hline Entrance & & & & & & \\
\hline Triage & & & & & & \\
\hline Trauma & & & & & & \\
\hline Febrile & & & & & & \\
\hline General room & & & & & & \\
\hline Intensive care unit & 4.01 & 4.02 & 4.03 & 4.04 & 4.04 & 4.05 \\
\hline Entrance & & & & & & \\
\hline Single room 1 & & & & & & \\
\hline Single room 2 & & & & & & \\
\hline General room 1 & & & & & & \\
\hline General room 2 & & & & & & \\
\hline
\end{tabular}

Note: Green indicates the highest score (5-6), yellow indicates a moderate score (3-4) and red indicates the lowest score (1-2).

Table 5: C5: Legibility of place.

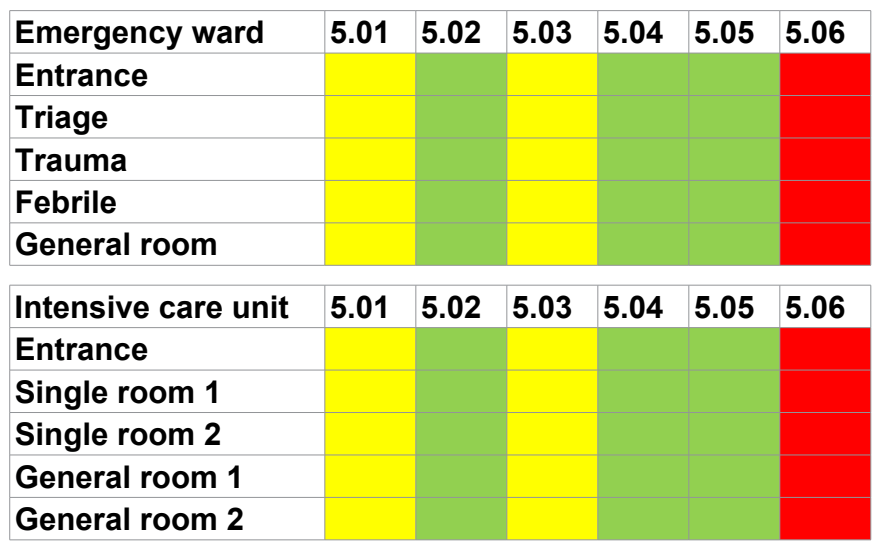

Note: Green indicates the highest score (5-6), yellow indicates a moderate score (3-4) and red indicates the lowest score (1-2).

\section{C4: Comfort and control}

The emergency ward and ICU lighting was adjusted with a button that was not easily accessible to the patients (Table 4). Lights in the wards had no dimmer function, and there were no bedside lamps. Nurses used flashlights on mobile phones to perform different nursing activities, such as blood sampling. Moreover, the emergency ward closed the doors to the febrile and trauma rooms ( $2 \times 3$ beds) to reduce unwanted sounds; however, shared rooms affected the noise level. The ICU's single rooms offered a quiet and calm environment when the door was closed.

In general, in the rooms ( 3,4 beds) in the ICU, minimization of sound was not feasible. However, the noise level was lower compared to the emergency ward's larger general room (14 beds), and noise from the busy outdoor traffic situation when the doors were opened contributed to a high noise level. In addition, the temperature was regulated by health professionals in the wards by adjusting ceiling fans or air-conditioning from the front desk.

\section{C5: Legibility of place}

Signs from outside of the hospital to find the way to the emergency ward were limited, so it was difficult for patients and relatives to easily find the ward (Table 5). However, the emergency ward was structured in a logical
Table 6: C6: Interior appearance.

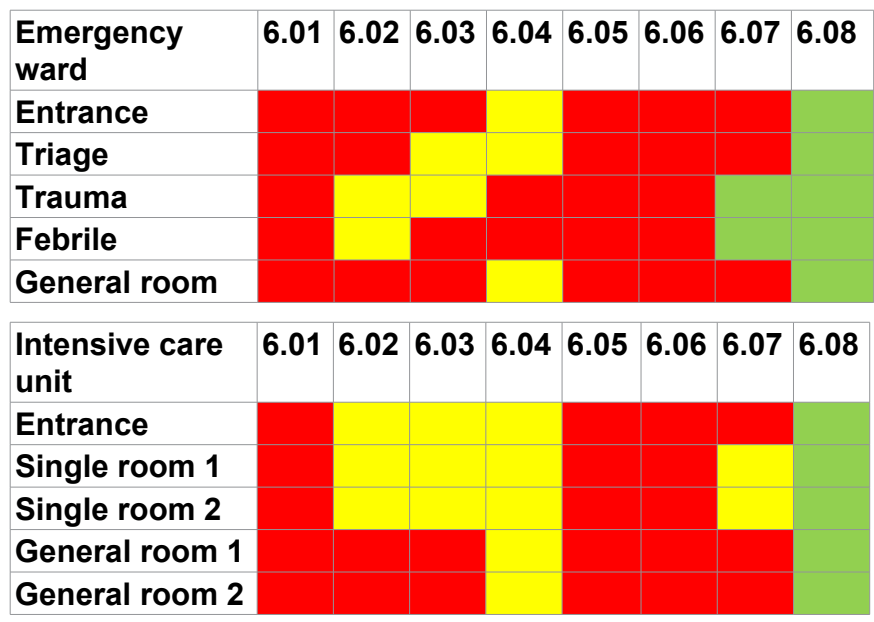

Note: Green indicates the highest score (5-6), yellow indicates a moderate score (3-4) and red indicates the lowest score (1-2).

Table 7: C7: Facilities.

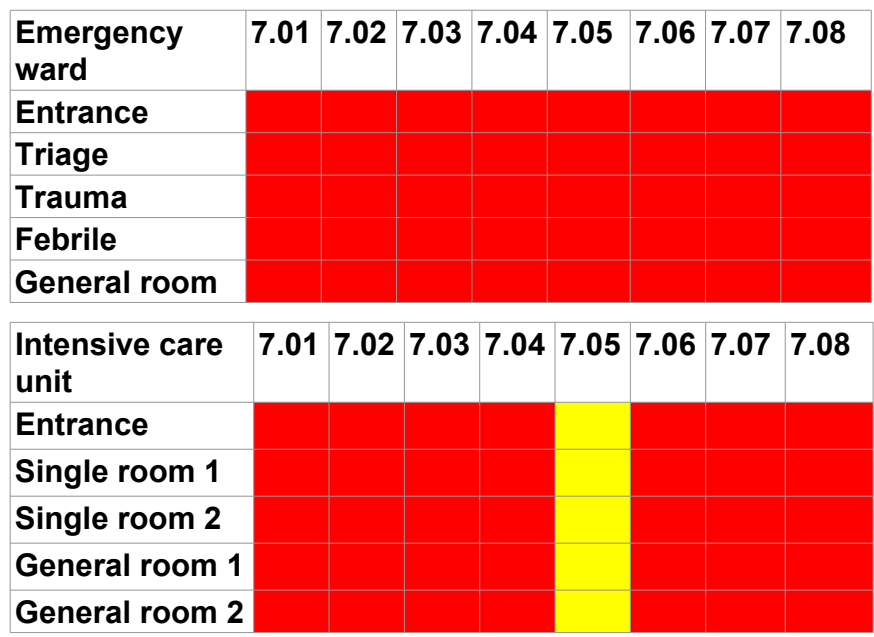

Note: Yellow indicates a moderate score (3-4) and red indicates the lowest score (1-2).

way with clear signposts, and it was easy to get in touch with staff members at the ward level. The emergency ward was also in close proximity to the overall emergency department, a small department, which made it easy to find. However, there was a lack of places solely for staff members, which allowed unauthorized access by patients or relatives to these areas (for example, beyond the front desk, nurses' office).

\section{C6: Interior appearance}

In the emergency ward, ants were noted in several places (Table 6). However, the patients' rooms showed no visible dirt (for example, floor and equipment). Moreover, the interior appearance was bright but not airy, and the environment had limited free areas compared to the ICU, which had more free areas that allowed staff to move freely between and inside rooms. The ICU was clean with no visible ants in the patient rooms or beds. In addition, the environment in the wards had limited spaces to store personal belongings, such as wardrobes and safety deposit boxes.

\section{C7: Facilities}

There was no available toilet at the ward level, so 
Table 8: C8: Staff.

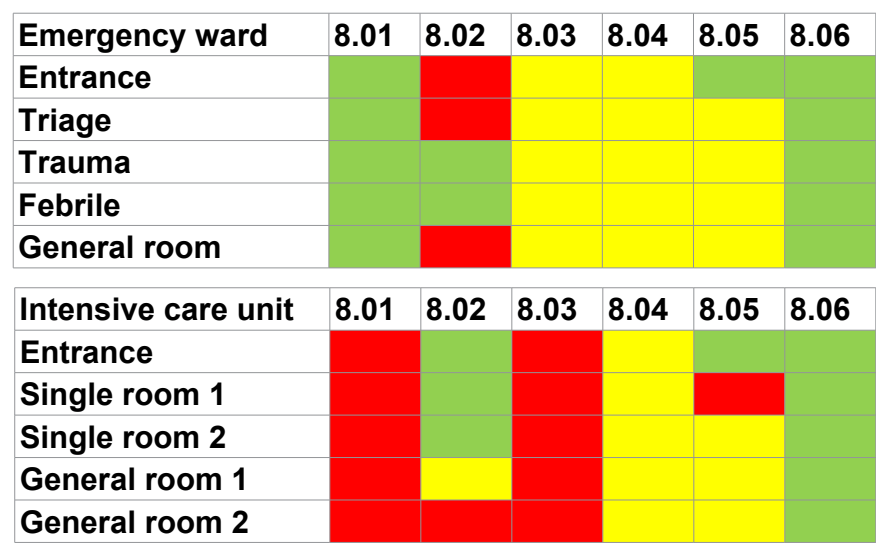

Note: Green indicates the highest score (5-6), yellow indicates a moderate score (3-4) and red indicates the lowest score (1-2).

patients and relatives had to visit available toilets at the hospital level (Table 7). This toilets were difficult to find (no signs), difficult to access and had limited space in the hospital (i.e., they were not adapted for disabled patients). Furthermore, there were a limited number of water machines in the wards (emergency ward $=2$, ICU $=1$ ) as well as limited access (for example, in the ICU, the water machine was located in the staff room).

\section{C8: Staff}

The wards were crowded, especially the emergency ward, with a large number of patients to care for (ICU $=9$, emergency $=20$ ) as well as relatives and a high number of staff members (emergency $=35, \mathrm{ICU}=20$ ) (Table 8). Therefore, it was difficult for staff to work undisturbed. Moreover, the wards had few computers (emergency $=4, I C U=2$ ) to use in relation to daily work. The working environment was busy, and several staff members simultaneously spoke and worked side by side. In addition, the wards were overloaded with patients and their relatives, so staff members were often interrupted during work, especially in the general care rooms. In the smaller rooms, such as the febrile and trauma rooms at the emergency ward as well as the single room at the ICU, staff had better opportunities to work undisturbed because there was a door to close as well as a limited number of patients to care for in the rooms. A lack of doors as well as the busy area of the front desks placed near or within patient rooms made it difficult for staff to work without interruption. Furthermore, the staff rooms in the wards were small in relation to the number of staff (emergency $=35, I C U=$ 20) and dirty, with no place to store personal belongings (wardrobes, safety deposit boxes). Private bags were placed on a shared table. There were a few small toilets for staff with no locks.

\section{Discussion}

The aim of this study was to investigate the care environment of emergency care (emergency ward and intensive care unit) at one private teaching hospital in Vietnam. According to Nightingale [5], the care environment, including fresh air and inspiring views, is important for health and well-being. However, this study found the opposite. The hospital's caring environment was described as stressful, with heavy traffic and crowded wards that were not calming or interesting for patients, relatives and staff. The dominant colour in the results was red with regard to ASPECT's [13] eight areas. This result may be related to a lack of resources due to overcrowded wards. This result is in line with earlier research focusing on patients' dissatisfaction with poor environments in hospitals $[2,8,19]$. The current study also found a busy outdoor environment as well as indoor views of hospital corridors instead of inspiring views of nature, which can provide refreshing rehabilitation. The current study found a lack of available natural areas, which can have a calming effect on patients [19]. Furthermore, no daylight was available in the ICU (windows in corridors), which Nightingale [5] emphasizes as important because dark rooms can be perceived as gloomy. A lack of bedside lamps or the ability to adjust the temperature influence the quality of sleep and stress.

According to research $[3,10,19]$, it is important that the care environment is adapted to provide the best conditions for patients' recovery by using single rooms equipped with doors for a peaceful and quiet environment. However, this was not the case for the emergency ward: 20 patients shared beds and were treated in a small area with limited space for private belongings, and other patients and relatives could view care activities due to the lack of doors. Furthermore, constant and sometimes sharp or sudden noise from monitors is disturbing and has a negative impact on health because physical environment is important to create a healing environment $[20,21]$. Therefore, nurses have a significant responsibility to improve the healthy working environment by using evidence-based nursing for well-being and better health $[5,9]$. According to research $[3,10]$, it is important that the care environment is adapted to provide the best conditions for patients' recovery as well as the possibility of relaxation for staff at work. Therefore, it is important to improve the indoor views and environment, such as through the use of air improvement tools, colours and drawings on the walls, better signage to find the way to the hospital, and clean ward areas and toilets. A lack of available toilets at the ward level means that patients must leave wards to visit the toilet. This is concerning because the majority of patients are critically ill [22]. This necessary movement could be risky because a fall could cause a fracture and worsen the patient's state of health, which is a health hazard for critically ill patients [22]. Moreover, dirty toilets with no locks that are inaccessible to disabled people decrease health and well-being among patients, relatives and staff $[2,4]$.

Moreover, the lack of meal service for patients is highlighted as a limitation of the care environment 
due to limited resources at Vietnamese hospitals [2]. Relatives commonly assist patients with food in the hospital. Therefore, available water machines are important within the ward area, as are nurses who are committed to improving the quality of care through nutrition. III health worsens with malnutrition, so meal services are important to improve the quality of care. In addition, research [19] stresses the significance of patients having family members close to them to improve the psychosocial environment within hospital care. For example, the current study found that relatives had to be outside the entrance due to limited space in the emergency ward, which is an inadequate way of using limited resources because the Vietnamese healthcare system depends on relatives' involvement in basic healthcare due to the limited number of staff members.

In addition, research [1] shows that patients choose private caregivers to receive a high quality of care. Therefore, it is concerning that the Vietnamese healthcare environment is inadequate in giving patients the care they pay for. Nguyen, et al. [4] suggest that healthcare in Vietnam would benefit from a review by a health authority to ensure the quality of care regarding differences in public and private hospitals in Vietnam. Patient safety is an important task in nursing that contributes to health and well-being [9]. Health professionals have the opportunity to improve patients' healthcare environment by providing fresh air and bedside lamps, allowing patients to regulate the temperature, and using curtain, drapes, and doors for increased privacy and integrity to improve the quality of care $[20,21]$. In summary, the present study shows that the care environment for critically ill patients requires improvement to stimulate recovery and well-being $[19,22]$.

\section{Limitations}

A limitation of this study was the small sample size due to limited time (six days) for data collection (observations $=652$, field notes $=$ nine $A 4$ pages). The reason was limited timetable due to content of courses within nursing programme (structure to conduct a bachelor thesis) as well as students possibility to study nursing abroad. Moreover, this study was conducted at one emergency department (emergency ward and ICU) at one private teaching hospital in one country, which limits the ability to generalize the results to other specialties, departments, and hospitals. However, the study used a well-established protocol to measure staff and patient environment calibration, the toolkit ASPECT [13], which contributes to the validity and reliability of the study. Moreover, the credibility of the study is satisfactory because different data collection methods were used (so-called triangulation) to gain a broader understanding of the care environment. The field notes collected during the study represented reflections on the observations, enabling a more comprehensive understanding of the observations. The field notes were analysed using a well-known content analysis method [15]. In Addition, Swedish rules and guidelines [17,18] support student's thesis, even when data collection is performed in another country IF no negative effects for the participants is shown. Moreover, countries as Sweden and Vietnam have different social structures and education and healthcare systems, which must be taken into consideration with regard to the transferability of the current study. Further studies are needed to develop knowledge regarding the care environment to improve the quality of care within hospital settings.

\section{Conclusion}

This study generates knowledge regarding the care environment for critically ill patients. Nurses have an important role in increasing well-being and recovery by improving patient safety, and a healthy environment is a significant part of this improvement. Patients need less crowded caring environments for improved health and well-being. This includes clean rooms, beds that are curtained and not shared, and low talking and music to decrease strong and/or sudden sounds. These are inexpensive interventions and improvements. Increased awareness is also needed among caregivers and decision makers regarding the significance of a healthy care environment to improve the quality of care. A healthy care environment involves improvements to hospitals such as uncrowded rooms without unpleasant odours that are clean and have a comfortable temperature as well as water and meal services. Future research could consider nurses' experiences regarding adaptations of a healthy care environment within the framework of hospitals' limited resources.

\section{Acknowledgements}

We thank the managers at Hanoi Medical University who organized the data collection, and the staff at the included private teaching hospital who participated in the study and the University of Gothenburg Centre for Person-Centred Care (GPCC), Gothenburg, Sweden.

\section{Funding}

The authors received no financial support for this research or for the authorship of and/or publication of this article.

\section{Conflicting Interests}

The authors declare no conflict of interest.

\section{References}

1. Löfgren C (2014) Catastrophic Health Expenditure in Vietnam: Studies of Problems and Solutions (Medical Dissertation, 1673). Umeå: Department of Public Health and Clinical Medicine Epidemiology and Global Health, Umeå University.

2. Thanh Cong N, Thu Tuyet Mai N (2014) Service quality and its 
impact on patient satisfaction: An investigation in Vietnamese Public Hospitals. J Emerg Econ Islam Res 2: 1-13.

3. Van Huy N, Dung NN, Thang CD, Hanh LT (2018) Patient satisfaction with health care services at a national institute of ophthalmology. Int J Health Plann Manage 33: e251-e262.

4. Nguyen Thi PL, Lê TG, Empereur F, Briancon S (2002) Satisfaction of Patients Hospitalized in Ho Chi Minh City, Vietnam. Sante Publique 14: 345-360.

5. Nightingale F (1860) Notes on Nursing - What It Is, and What It Is Not. Cambridge Library Collection, Cambridge.

6. World Health Organization, WHO (2011) A health financing review of Vietnam with a focus on social health insurance. Genève: World Health Organization, Geneva.

7. World Health Organization, WHO. Viet Nam.

8. Nguyen MP, Wilson A (2017) How could private healthcare better contribute to healthcare coverage in Vietnam? Int J Health Policy Manag 6: 305-308.

9. International Council of Nurses (2012) The ICN Code of Ethics for Nurses. Geneva, Switzerland.

10. Selanders LC (2010) The power of environmental adaptation: Florence Nightingale's original theory for nursing practice. J Holist Nurs 28: 81-88.

11. Hanoi Medical University, HMU (2007) History of Hanoi Medical University.

12. Hanoi Medical University (2014) Training capacity of Hanoi Medical University.

13. National Health Service, NHS (2008) A Staff and Patient
Environment Calibration Toolkit (ASPECT) Instructions, scoring and guidance.

14. Polit D, Beck CT (2012) Nursing research: Generating and assessing evidence for nursing practice. Wolters Kluwer Health/Lippincott Williams \& Wilkins, Philadelphia.

15. Graneheim UH, Lundman B (2004) Qualitative content analysis in nursing research: Concepts, procedures and measures to achieve trustworthiness. Nurse Educ Today 24: 105-112.

16. Gilliland D (2012) Microsoft Office Excel 2007/2010. OfficePro 72: 44-45.

17. Codex Rules \& guidelines for research (2018) The humanities and social sciences.

18. Swedish Code of Statutes. 1982:763. Hälso- och sjukvårdslagen (Health and Medical Services Act).

19. Sloan Devlin A, Campos Andrade C, Carvalho D (2016) Qualities of inpatient hospital rooms. Patients perspectives. HERD 9: 190-211.

20. Abbas MY, Ghazali R (2011) Physical Environment: The major determinant towards the creation of a healing environment? Procedia - Social and Behavioral Sciences 30: 1951-1958.

21. Ghazali R, Abbas MY (2011) Paediatric wards: Healing environment assessment. Asian Journal of Environment Behavior Studies 2: 64-76.

22. Centre for Clinical Practice at NICE (2007) Acutely ill patients in hospital: Recognition of and response to acute illness in adults in hospital. National Institute for Health and Clinical Excellence, London, UK. 